\title{
PENSi: Núcleo de Pesquisa em Engenharia Semiótica e Interação
}

\author{
Raquel O. Prates \\ Departamento de Ciência da Computação \\ Universidade Federal de Minas Gerais (UFMG) \\ Belo Horizonte, MG, Brasil \\ rprates@dcc.ufmg.br
}

\begin{abstract}
RESUMO
Este resumo estendido tem por objetivo apresentar o PENSi (Núcleo de Pesquisa em Engenharia Semiótica e Interação) do Departamento de Ciência da Computação da UFMG, com foco nas suas pesquisas mais recentes.
\end{abstract}

\section{PALAVRAS-CHAVE}

Pesquisa em IHC, Engenharia Semiótica, Interação, Comunicabilidade, Sistemas Colaborativos, Acessibilidade, Educação, Métodos de Avaliação.

\section{APRESENTAÇÃO GERAL}

O Núcleo de Pesquisa em Engenharia Semiótica e Interação, conhecido como PENSi, é um grupo de pesquisa na área de IHC do Departamento de Ciência da Computação (DCC) da UFMG. O grupo foi criado em 2006, com a chegada da Profa. Raquel Oliveira Prates ao DCC/UFMG. Desde então o grupo tem realizado pesquisa na área de IHC, tendo como sua principal fundamentação teórica a teoria da Engenharia Semiótica [1]. Dentre os diversos temas que são foco na pesquisa do PENSi estão: a própria teoria da Engenharia Semiótica, incluindo modelos para apoio a projeto de sistemas interativos e métodos de avaliação de comunicabilidade, sistemas colaborativos, programação por usuário final (End-User Development), Educação de IHC e de Computação, Jogos, Novos domínios de interação e também aspectos relacionados ao impacto da tecnologia e responsabilidade social associada à criação destes sistemas.

O PENSi é um dos diversos laboratórios de pesquisa do DCC/UFMG. Em termos de infraestrutura oferece aos seus membros um laboratório contendo estações de trabalho e um pequeno espaço para reuniões. Além disso, o PENSi é um dos laboratórios responsáveis pelo laboratório de teste com usuários do

Permission to reproduce or distribute, in whole or in part, material extracted from this work, verbatim, adapted or remixed, as well as the creation or production from the content of such work, is granted without fee for non-commercial use, provided that the original work is properly credited.

IHC 2019 - TRILHA FÓRUM DOS GRUPOS DE PESQUISA, Outubro 21-25,

2019, Vitória, Brasil. In Anais Estendidos do XVIII Simpósio Brasileiro sobre Fatores Humanos em Sistemas Computacionais. Porto Alegre: SBC.

(C) 2019 by the author(s), in accordance with the terms of the Creative Commons Attribution-NonCommercial 4.0 International Public License (CC BY-NC 4.0).
DCC. Os membros do PENSi contam também com toda a infraestrutura oferecida à comunidade acadêmica do DCC e da UFMG, de forma geral.

Neste resumo, apresentamos brevemente as linhas de pesquisa do PENSi, os temas de pesquisa sendo explorados mais recentemente, assim como seus principais resultados e uma visão geral de quem são participantes e colaboradores do grupo.

\section{LINHAS DE PESQUISA}

Conforme mencionado, o PENSi tem desenvolvido pesquisa em várias linhas, sendo que, com frequência, temas de pesquisa envolvem duas ou mais destas linhas. Assim, vamos apresentar brevemente estas linhas, e depois explicamos algumas das pesquisas recentes do grupo.

De forma geral, as principais linhas de pesquisa em que o PENSi tem atuado podem ser descritas como:

- Teoria da Engenharia Semiótica (EngSem): A EngSem foi formalizada como teoria a partir do livro de de Souza sobre a mesma em 2005 [1]. Assim, as pesquisas do PENSi sobre a teoria buscam investigar a aplicabilidade e consolidação da teoria e seus modelos e métodos a diferentes contextos. Assim, como propor novos modelos ou métodos, ou sua adaptação para contextos em que se perceba sua necessidade

- Sistemas Colaborativos: A pesquisa sobre Sistemas Colaborativos foca principalmente em aspectos relacionados com a interação colaborativa, assim como modelos e métodos que apoiem o projeto ou avaliação desta.

- Desenvolvimento pelo Usuário Final (End-User Development): Nesta linha colocamos toda a pesquisa que está associada a como o usuário final pode se apropriar da tecnologia, adaptando-a seu contexto. Assim, o foco vai desde permitir a customização do sistema pelo usuário final, até modelos e sistemas que permitam que o usuário final crie novas instâncias ou tipos de interação nestes sistemas.

- Educacão: Em relação à educação, o foco na área de pesquisa tem envolvido diferentes aspectos: educação de IHC e ensino de computação, com especial foco no ensino pensamento computacional no ensino fundamental II (que utiliza como 
base as Metáforas de Perspectiva Cultural fundamentadas na EngSgem).

- Domínios e Tecnologias Específicos: Nesta linha temos investigado questões de interação que consideram especificidades de domínios ou de tecnologias. Uma das áreas em que temos atuado constantemente é a de jogos. Além disso, vale ressaltar a pesquisa em domínios específicos como interação com chatbots ou com tecnologias associadas à morte.

- Responsabilidade Social: A tecnologia de informação tem cada vez mais se integrado ao cotidiano das pessoas. Assim, vários aspectos relacionados ao uso que as pessoas fazem ou podem fazer desta tecnologia e em diferentes momentos de sua vida tem sido foco da nossa pesquisa. Em especial, o PENSi tem ao longo dos anos feito pesquisa na linha de acessibilidade, e como permitir um acesso mais amplo à tecnologia. Além disso, recentemente temas como privacidade em redes sociais, explicabilidade de algoritmos e gerenciamento do legado digital pós-morte têm também sido contemplados.

Vale ressaltar que mesmo na pesquisa nas linhas que não são diretamente relacionadas à Engenharia Semiótica, a teoria tem um papel relevante fundamentando a o entendimento dos fenômenos de interação e no uso de métodos de modelos para a realização das pesquisas.

Na próxima seção apresentamos alguns dos temas de pesquisa mais recentes que têm sido investigados no PENSi. Note-se que vários deles envolvem mais de uma linha de pesquisa.

\section{TEMAS DE PESQUISA RECENTES}

Os temas de pesquisa recentes abordados diferentes tópicos associados às linhas de pesquisa. A pesquisa sobre privacidade em redes sociais, está associada tanto a Responsabilidade Social, na medida que investiga o impacto social da divulgação de informações através das redes sociais. Além disso, a pesquisa propõe um modelo (MDP [2]) que tem por objetivo apoiar o projetista nas decisões sobre aspectos de privacidade nestes sistemas.

Um modelo também voltado para apoiar o projetista em decisões em tempo de design foi o SIGMa [3]. O foco deste modelo é no apoio ao projetista a decisões sobre cenários futuros que podem ser gerados durante o uso do sistema, seja por possibilidades de configuração ou mudanças que o sistema possa oferecer ao usuário, seja pela evolução da interação no tempo. No contexto de interações futuras temos investigado também aspectos relacionados a que aspectos são relevantes de se oferecer para o usuário, para que ele possa antecipar corretamente os efeitos de suas decisões, que só ocorrerão em outro momento no tempo [4]. Um contexto em que esta percepção é fundamental é em sistemas associados ao pósmorte, e.g. gerenciamento de legado digital [5].

Permitir que usuários customizem e adaptem sistemas para seus contextos é cada vez mais essencial, considerando-se a grande quantidade de sistemas que as pessoas usam no seu cotidiano e nos mais diversos contextos. Na área de saúde e sistemas que apoiam tratamentos, isso pode se tornar fundamental, uma vez que mesmo pacientes sendo tratados pelas mesmas condições clínicas têm necessidades individuais diferentes[6][7]. Nesta direção, uma linha que está no nosso horizonte é na criação de ambientes em que usuários finais, como terapeutas possam ir além da customização do sistema, mas possam criar novas instâncias de jogos ou sistemas interativos específicos. Entendemos que esta necessidade se aplica não apenas à área de saúde, mas também para a educação.

$\mathrm{Na}$ linha de educação, um foco que temos investigado é justamente o ensino de IHC. Como a disciplina tem sido ensinada no Brasil, e quais são as questões nas quais a comunidade pode investir para apoiar mais este ensino [8]. No contexto atual, o ensino de Pensamento Computacional no nível básico da educação também tem sido um foco de investigação. Nesta pesquisa, embora o foco não seja a área de IHC, temos aplicado conhecimento gerado em IHC (e.g. Metáforas de Perspectivas Culturais) para explorar o contexto de ensino [9].

O foco na ampliação do uso da tecnologia pela sociedade tem nos levado a investigar também temas associados à acessibilidade. No caso, não apenas na identificação e geração de tecnologias para usuários com necessidades especiais, mas também em como métodos da EngSem podem ser adaptados para que levem em conta também a comunicabilidade sob o ponto de vista de usuários que apenas tem acesso a uma versão intermediada da tecnologia [10].

Os avanços na própria tecnologia e a ampliação de domínios e usos que têm sido feitos da mesma, geram novas questões relevantes para a interação e impacto da tecnologia. Nesta linha, temos explorado temas associados a estes novos contextos. Em relação a novas interações entre as pessoas criadas pela tecnologia, temos investigado a confiabilidade de sistemas de economia colaborativa [11], sistemas que têm por objetivo criar permitir parcerias e interações entre pessoas que fazem parte de uma mesma comunidade [12], e estratégias para curadoria coletiva de conteúdo científico [13]. Além disso, cada vez mais chatbots têm sido criados para apoiar a interação de pessoas com informação ou empresas, e como apresentar para os usuários as interações possíveis deste agente conversacional, nem sempre é simples [14]. Finalmente, algoritmos cada vez mais complexos têm gerado resultados cujo objetivo é influenciar as decisões ou as ações das pessoas, então torna-se fundamental que estas pessoas entendam as recomendações ou informações que recebem, e a questão de explicabilidade destes sistemas.

Finalmente, nossas pesquisas partem da premissa da EngSem da interface como uma comunicação do projetista para seus usuários e usam este quadro teórico para propor novos modelos [2][3] ou adaptações para métodos[10]. Além disso, nossas pesquisas com frequência exploram a aplicabilidade de modelos e métodos da EngSem para contextos mais amplos ou específicos do que aqueles para os quais foram propostos, contribuindo direta [15][16]ou indiretamente para a consolidação destes métodos ou modelos. 


\section{RESULTADOS}

Ao longo dos anos, os principais resultados científicos do PENSi têm sido publicados na forma de artigos em eventos e periódicos (atualmente são mais de 100 artigos publicados). Muitos destes artigos apresentam resultados de pesquisas de mestrado (25 mestre formados, contando aqueles com foco em IHC, e em temas interdisciplinares) e doutorado ( 2 doutores formados), sem contar a participação de mais de 50 alunos de graduação colaborando através de iniciação científica ou projetos de conclusão de curso. Várias destas pesquisas resultaram também em sistemas ou protótipos que foram apresentados à comunidade: como por exemplo o SMART (ferrramenta para apoiar o uso da Manas), o PryMeVis (para apoiar o uso do MDP), ou o Sêntimus (jogo para crianças com hipersensibilidade sonora.

Além disso, o PENSi e seus membros buscam também a geração de materiais didáticos que possam ser úteis no ensino, seja de conteúdos de IHC diretamente (e.g. Engenharia Semiótica e seus métodos), seja em temas voltados para aspectos sociais da tecnologia (e.g. tecnologias pós-mortem) ou que sejam necessários para a pesquisa na área de IHC ou com usuários (e.g. métodos de pesquisa qualitativos). Temos tentado também criar projetos de extensão em que as pesquisas possam trazer um benefício direto para a comunidade, além de sua contribuição científica (e.g. ensino de pensamento computacional no ensino fundamental II em parceria com o Centro Pedagógico, escola de ensino fundamental da UFMG).

\section{PARTICIPANTES E COLABORADORES}

Estes resultados têm sido alcançados aos longos dos anos com o trabalho dos membros do PENSi e seus muitos colaboradores. Atualmente o PENSi conta com a participação, além da sua coordenadora - Profa. Raquel, com 5 alunos de doutorado, 10 de mestrado, 4 de iniciação científica e 4 de trabalho de conclusão de curso. Os colaboradores do PENSi contribuem com a pesquisa do grupo sob diversas perspectivas, enriquecendo-a. Como seria impossível citar nominalmente todos eles, apresentamos de forma geral a origem e natureza da colaboração. Vários colaboradores são professores de outras áreas do próprio DCC, que atuam em colaboração em projetos de pesquisa ou co-orientações de alunos. Professores de outras áreas e departamentos da UFMG, como Música, Artes, Sociologia, Ciência da Informação e Estatística, permitem uma abordagem interdisciplinar de vários temas. Alunos egressos do PENSi que são pesquisadores de outras instituições de Minas Gerais, como UFVJM, IFMG-Campus Formiga, CEFETMG e UFSJ são colaboradores constantes do PENSi. Finalmente, interagimos com diversos pesquisadores da comunidade de IHC nacional e internacional. Na comunidade nacional, contamos com colaboradores de longa data como colegas do SERG e IDEIAS (PUC-Rio) e PUCRS, outros, mais recentes, com os quais identificamos parcerias de interesse ao longo dos anos de atuação do PENSi (muitas vezes através de nossa participação no IHC) como IBM-Research, UFMT, UFLA, UFAM, UFPA, PUC-Minas, para citar alguns. Finalmente, PENSi busca interações com pesquisadores de IHC da comunidade internacional, como por exemplo a parceria com o Center for Human-Computer Interaction da Penn State University. Certamente a contribuição de todos os membros (egressos e atuais) e colaboradores do PENSi são fundamentais nos resultados obtidos pelo grupo.

\section{REFERÊNCIAS}

[1] Clarisse S. De Souza. 2005. The semiotic engineering of human-computer interaction. MIT press, Cambridge, Massachusetts.

[2] Maria Lucia B. Villela. Um modelo de design de privacidade para o compartilhamento de informações pessoais em redes sociais online. Tese de doutorado, DCC/UFMG, 2016.

[3] Manoel Pereira Junior. Um Modelo para Apoiar Projetistas de Sistemas Colaborativos na Antecipação de Cenários. Tese de doutorado, DCC/UFMG, 2016. .

[4] Raquel O. Prates, MaryBeth Rosson, and Clarisse S. de Souza. 2015. Interaction Anticipation: Communicating Impacts of Groupware Configuration Settings to Users. In End-User Development, Paloma Díaz, Volkmar Pipek, Carmelo Ardito, Carlos Jensen, Ignacio Aedo, and Alexander Boden (Eds.). Lecture Notes in Computer Science, Vol. 9083. Springer International Publishing, Cham, 192--197. https://doi.org/10.1007/978-3-319-18425-8 15

[5] Fabrício H. S. Pereira, Raquel O. Prates, Cristiano Maciel, and Vinícius C. Pereira. 2016. Analysis of Interaction Anticipation and Volitive Aspects in Digital Posthumous Communication Systems. In Proceedings of the 15th Brazilian Symposium on Human Factors in Computing Systems (IHC '16). ACM, New York, NY, USA, Article 19, 10 pages. DOI: https://doi.org/10.1145/3033701.3033720

[6] Henrique Rossi; Raquel Prates; Sibele Santos; Renato Ferreira, 2019.Development of a Virtual Reality-Based Game Approach for Supporting Sensory Processing Disorders Treatment. Information, 10(5), 177. http://dx.doi.org/10.3390/info10050177.

[7] Débora Line Gomes, Cybelle Loureiro, Raquel Prates. SÊNTIMUS: um Jogo Digital Musical para Crianças com Hipersensibilidade Sonora e Características dos Transtornos do Neurodesenvolvimento. Anais Estendidos do XIX Simpósio Brasileiro de Computação Aplicada à Saúde. Porto Alegre: Sociedade Brasileira de Computação, 2019 . p. 79-84.: https://doi.org/10.5753/sbcas.2019.6288.

[8] Tatiane Gomes Guimarães; Raquel Oliveira Prates. HCI Education in Brazil in the light of Curricula Guidelines. In: 2018 XLIV Latin American Computer Conference (CLEI). IEEE, 2018. p. 784-793.

[9] Gabriela A. Araujo de Oliveira, Oto Braz Assunção, and Raquel Oliveira Prates. 2019. Using Cultural Viewpoint Metaphors in the Analysis of Computational Thinking Teaching. In Proceedings of the 18th Brazilian Symposium on Human Factors in Computing Systems (IHC 2019), October 22-25, 2019, Vitoria - ES, Brazil. ACM, New York, NY, USA, 13 pages. https://doi.org/10.1145/3357155.3358466.

[10] Lucas Pedroso Carvalho, Raquel Oliveira Prates, and André Pimenta Freire. 2019. A Proposal to Adapt the Semiotic Inspection Method to Analyze Screen Reader Mediated Interaction. In Proceedings of the 18th Brazilian Symposium on Human Factors in Computing Systems (IHC 2019), October 22-25, 2019, Vitória - ES, Brazil. ACM, New York, NY, USA, 11 pages. https://doi.org/10.1145/3357155.3358455.

[11] Geanderson E. dos Santos and Raquel O. Prates. 2018. Evaluating the PROMISE framework for Trust in Sharing Economy System. In Proceedings of the 17th Brazilian Symposium on Human Factors in Computing Systems (IHC 2018). ACM, New York, NY, USA, Article 20, 11 pages. DOI: https://doi.org/10.1145/3274192.3274212

[12] Luiz Paulo Damilton Corrêa. EstudaJunto UFMG: Um Aplicativo Móvel de Encontros e Coprodução para Estudos na Universidade. Dissertação de Mestrado, DCC/UFMG, 2017.

[13] Lídia Silva Ferreira and Raquel Oliveira Prates. 2019. Investigando curadoria coletiva em um contexto científico de compartilhamento de métodos e modelos. Workshop de Teses e Dissertações (WTD) do IHC'19: XVIII Simpósio Brasileiro sobre Fatores Humanos em Sistemas Computacionais (IHC'19), 4 pags

[14] Francisco A. M. Valério, Tatiane G. Guimarães, Raquel O. Prates, and Heloisa Candello. 2017. Here's What I Can Do: Chatbots' Strategies to Convey Their Features to Users. In Proceedings of the XVI Brazilian Symposium on Human Factors in Computing Systems (IHC 2017). ACM, New York, NY, USA, Article 28, 10 pages. DOI: https://doi.org/10.1145/3160504.3160544

[15] Erica Rodrigues de Oliveira and Raquel Oliveira Prates. 2018. Intermediated Semiotic Inspection Method. In Proceedings of the 17th Brazilian Symposium on Human Factors in Computing Systems (IHC 2018). ACM, New York, NY, USA, Article 29, 10 pages. DOI: https://doi.org/10.1145/3274192.3274221

[16] Ana Paula de Carvalho, Fabrício Horácio Sales Pereira, Oto Braz Assunção, Amanda Fernandes Pereira, and Raquel Oliveira Prates. 2019. An Analysis of MoLIC's Consolidation. In Proceedings of the 18th Brazilian Symposium on Human Factors in Computing Systems (IHC '19), October 22-25, 2019, Vitoria - ES, Brazil. ACM, New York, NY, USA, 15 pages. https://doi.org/10.1145/3357155.3358461 